\title{
The relationship between cervical precancerous lesion galectin-3 and p27 protein expression and clinical prognosis
}

\author{
JIANTING MA, XINGGUANG ZHANG, GANG HE and CHUNLIN YANG \\ Department of Obstetrics and Gynecology, Yuyao People's Hospital, Yuyao, Zhejiang 315400, P.R. China
}

Received July 13, 2017; Accepted October 31, 2017

DOI: $10.3892 / \mathrm{ol} .2017 .7503$

\begin{abstract}
The relationship between galectin-3 and $\mathrm{p} 27^{\mathrm{kip} 1}$ protein expression levels in cervical precancerous lesions and clinical prognosis were studied. A total of 74 patients with cervical intraepithelial neoplasia [(CIN), 20 cases classified as stage I, 24 cases as stage II and 30 cases as stage III] were enrolled in this study. Tissue galectin-3, p2 $7^{\mathrm{kip} 1}$, vascular endothelial growth factor (VEGF)-2 and cyclin D protein levels were detected via immunohistochemical staining and reverse transcription polymerase chain reaction (PCR). Follow-up median duration was 13.5 months and recurrence rate was determined. Galectin-3, VEGF-2, and cyclin D expression was elevated in patients with higher stage CIN, whereas $\mathrm{p} 27^{\mathrm{kip} 1}$ showed the opposite trend $(\mathrm{p}<0.05)$. During follow-up, there were 3 cases $(15.0 \%)$ of recurrence in the CIN-I group, 5 cases $(20.8 \%)$ in the CIN-II group and 9 cases $(30.0 \%)$ in CIN-III the group. No significant difference in recurrence rate was noted among the groups $(\mathrm{p}>0.05)$. The upregulation of galectin-3 and downregulation of p27 ${ }^{\mathrm{kip} 1}$ in CIN tissues may be related to tumor progression. This phenomenon will require further verification.
\end{abstract}

\section{Introduction}

Cervical cancer has become a malignant tumor of importance. It threatens women's health, as its morbidity and mortality rates are second only to lung cancer, with a trend towards younger patients and increasing rates (1). It is generally believed that the occurrence of cervical cancer is related to high-risk human papilloma virus (HPV) infection and abnormal expressions of a variety of pathogenic genes (2). Galectin-3 is a member of lectin family and regulates intercellular adhesion, metastasis, angiogenesis, apoptosis, immune tolerance and other behaviors (3). Angiogenesis is a necessary

Correspondence to: Dr Jianting Ma, Department of Obstetrics and Gynecology, Yuyao People's Hospital, 800 East Road, Fengshan, Yuyao, Zhejiang 315400, P.R. China

E-mail: majianting1122@163.com

Key words: cervical intraepithelial neoplasia, galectin-3, p27 $7^{\mathrm{kip} 1}$, vascular endothelial growth factor-2, cyclin D condition for the in situ and metastatic growth of tumor cells, as well as an important factor for target organ localization and circulating blood metastasis (4). Markowska et al (5) confirmed that galectin-3 can activate $\mathrm{N}$-acetylglucosamine transferase $\mathrm{V}$, leading to the phosphorylation of vascular endothelial growth factor (VEGF)-2 and promoting angiogenesis. Many scholars (6) believe that elevated expression of galectin-3 plays an important role in the occurrence and development of cervical cancer. However, some scholars hold the opposite opinion (7), pointing out that galectin-3 shows strongly positive expression in normal cervix and chronic cervicitis tissues, but lower levels in cervical precancerous lesions and invasive carcinomas. Therefore, the correlation between galectin-3 expression and cervical cancer occurrence needs in-depth analysis.

In addition, changes in the tumor cell cycle also play a key role in the occurrence of disease. The $\mathrm{p} 27^{\mathrm{kip} 1}$ protein is a multifunctional cyclin-dependent kinase inhibitor, which can stagnate cells in the G1 phase and reduce G1/S phase transition via the inhibition of cyclin D-CDK 4, cyclin E-CDK 2, and other complexes (8). p2 $7^{\mathrm{kip} 1}$ can also affect cell differentiation and apoptosis, thereby inhibiting cell proliferation (9). A number of studies (10) suggest that the downregulation of p $27^{\mathrm{kip} 1}$ may be a sensitive index for the early diagnosis and prognosis evaluation of cervical cancer.

This study therefore analyzed the relationship between galectin-3 and $\mathrm{p} 27^{\mathrm{kip} 1}$ protein expression in cervical precancerous lesion tissues and clinical prognosis, in order to provide a reference for the early diagnosis of disease.

\section{Materials and methods}

Patient data. A total of 74 patients diagnosed with cervical intraepithelial neoplasia (CIN) via hysteroscopic biopsy and pathology at Yuyao People's Hospital between January 2015 and June 2016 were continuously selected. There were 20 cases classified as CIN I, 24 cases as CIN II and 30 cases as CIN III. Patients in the CIN I group were aged 45-66 years (average, 51.2 \pm 8.6 years), with 12 cases of high-risk HPV infection. Patients in the CIN II group were aged 44-68 years (average, 53.2 \pm 7.9 years), with 16 cases of high-risk HPV infection. Patients in the CIN III group were aged 46-52 years (average, 52.6 \pm 6.8 years), with 20 cases of high-risk HPV infection. No differences in age and high-risk HPV infection rate were observed among the three groups $(\mathrm{p}>0.05)$. 
Informed consent was obtained from all patients in this study. Conization of cervix or radical operation was performed and radiotherapy and chemotherapy were not adopted.

Research methods. Galectin-3, p27 kip1, VEGF-2 and cyclin D expression was detected via immunohistochemical staining and reverse transcription polymerase chain reaction (RT-PCR). Tissue sections (5 $\mu \mathrm{m}$-thick) were made conventionally and stored at $-70^{\circ} \mathrm{C}$. Follow-up lasted for 6-22 months (median, 13.5 months) and recurrence rate was compared.

Immunohistochemical staining. Tissues were dewaxed using dimethylbenzene, hydrated using an ethanol gradient, and then were incubated in $3 \% \mathrm{H}_{2} \mathrm{O}_{2}$ for $20 \mathrm{~min}$ and then in normal goat serum working solution for $30 \mathrm{~min}$. Rat anti-human galectin-3, p27 ${ }^{\mathrm{kip} 1}, \mathrm{VEGF}-2$ and cyclin D monoclonal antibodies and $\beta$-actin (internal reference) primary antibody (working concentration of 1:2,000; Jiangsu Beyotime Institute of Biotechnology Co., Ltd., Jiangsu, China) were used to incubate tissues using a wet box at $4^{\circ} \mathrm{C}$ overnight. Following which, a rabbit anti-mouse IgG polyclonal secondary antibody (working concentration of 1:500; Jiangsu Beyotime Institute of Biotechnology) was used to incubate tissues using a wet box at $27^{\circ} \mathrm{C}$ for $20 \mathrm{~min}$. Finally, horseradish peroxidase-labeled streptavidin working solution (Jiangsu Beyotime Institute of Biotechnology) was used to incubate tissues using a wet box at $27^{\circ} \mathrm{C}$ for $20 \mathrm{~min}$, washed with phosphate-buffered saline (PBS), subjected to DAB staining and hematoxylin counter-staining, sealed with neutral gum, dried at room temperature and observed under an optical microscope. Staining results were evaluated using a semi-quantitative method according to stain intensity and positive cell rate. Dark brown-stained cytoplasm or nucleus was regarded as a positive indicator; no positive staining was scored as 0 points, weakly positive staining as 1 point, moderately positive staining as 2 points and strongly positive staining as 3 points. A positive cell rate $\leq 5 \%$ was scored as 0 points, $6-25 \%$ as 1 point, $26-50 \%$ as 2 points, $51-75 \%$ as 3 points and $>75 \%$ as 4 points. A sum between $0-3$ points was interpreted as negative and a sum between 4-12 points as positive.

$R T$-PCR. Total cellular RNA was extracted using TRIzol reagent and RNA concentration and purity were determined via ultraviolet spectrophotometer. cDNA was synthesized using a reverse transcription kit. Primers were produced by Shanghai Sangon Biotech Company according to Gene Bank sequences: Galectin-3 forward, 5'-GGTTTCATCCAGGAT CGAGCAGG-3' and reverse, 5'-ACAAAGATGGTCACGGT CTGCC-3', 445 bp; p27 ${ }^{\mathrm{kip} 1}$ forward, 5'-ACTACTTCTCCCGC CGCTAC-3' and reverse, 5'-GAAATCAAACAGAGGC CGCATG-3', 332 bp; VEGF-2 forward, 5'-TACCAGTGGAG GCCGACTTC-3' and reverse, 5'-GCACAAAGCGACTGGA TGAAC-3', 103 bp; cyclin D forward, 5'-TTCCTCTTCCTAC AGTACTC-3' and reverse, 5'-GCAACCAGCCCTGTCGT CTC-3', 399 bp; GAPDH forward, 5'-CGCGAGAAGATGAC CCAGAT-3' and reverse, 5'-GCACTGTGTTGGCGTAC AGG-3', 225 bp. The following reaction system mixture was employed: $2 \mu \mathrm{l}$ cDNA, $3 \mu \mathrm{l}$ forward primer, $3 \mu 1$ lower primer, $0.5 \mu \mathrm{l}$ Taq polymerase, $1 \mu \mathrm{l} \mathrm{dNTPs}, 3 \mu \mathrm{l} \mathrm{MgCl}_{2}, 5 \mu \mathrm{l} 10 \mathrm{X}$
Table I. Positive staining rate for each protein.

\begin{tabular}{lccccc}
\hline Group & Cases & $\begin{array}{c}\text { Galectin-3 } \\
\mathrm{n}(\%)\end{array}$ & $\begin{array}{c}\mathrm{p} 27^{\mathrm{kip} 1} \\
\mathrm{n}(\%)\end{array}$ & $\begin{array}{c}\text { VEGF-2 } \\
\mathrm{n}(\%)\end{array}$ & $\begin{array}{c}\text { Cyclin D } \\
\mathrm{n}(\%)\end{array}$ \\
\hline CIN I & 20 & $6(30.0)$ & $14(70.0)$ & $5(25.0)$ & $7(35.0)$ \\
CIN II & 24 & $9(37.5)$ & $11(45.8)$ & $10(41.7)$ & $12(50.0)$ \\
CIN III & 30 & $19(63.3)$ & $10(33.3)$ & $21(70.0)$ & $23(76.7)$ \\
$\chi^{2}$ & & 6.389 & 6.503 & 10.420 & 9.149 \\
P-value & & 0.041 & 0.039 & 0.005 & 0.010 \\
\hline
\end{tabular}

VEGF-2, vascular endothelial growth factor-2; CIN, cervical intraepithelial neoplasia.

buffer and water up to a final volume of $25 \mu \mathrm{l}$. Reaction conditions were as follows: $95^{\circ} \mathrm{C}$ for $5 \mathrm{~min}, 95^{\circ} \mathrm{C}$ for $30 \mathrm{sec}, 58^{\circ} \mathrm{C}$ for $30 \mathrm{sec}$ and $72^{\circ} \mathrm{C}$ for $60 \mathrm{sec}$ for 30 cycles and concluding with a termination cycle at $72^{\circ} \mathrm{C}$ for $10 \mathrm{~min}$. PCR products were identified via $2 \%$ agarose gel electrophoresis followed by UV imaging using a gel imaging analysis system and gray value analysis via digital photography.

Statistical analysis. SPSS 20.0 (SPSS, Inc., Chicago, IL, USA) software was used for statistical analysis. Measurement data were presented as mean \pm standard deviation (SD). One-way analysis of variance (ANOVA) was used for intergroup comparisons and LSD t-test was used for pairwise comparisons. Enumeration data were presented as cases or percentages (\%) and Chi-square test was used for intergroup comparisons. $\mathrm{P}<0.05$ was considered to indicate a statistically significant difference.

Ethics statement. The study was approved by the Ethics Committee of Yuyao People's Hospital and written informed consents were signed by the patients and/or guardians.

\section{Results}

Immunohistochemical staining. Positive expression rates for galectin-3, VEGF-2 and cyclin D increased in higher CIN-stage patients, but $\mathrm{p} 27^{\mathrm{kip} 1}$ expression presented the opposite result $(\mathrm{p}<0.05$; Fig. 1 and Table I).

$R T-P C R$. Galectin-3, VEGF-2 and cyclin D mRNA expression levels increased in higher CIN-stage patients, but p27 ${ }^{\mathrm{kip} 1}$ expression presented the opposite result ( $\mathrm{p}<0.05$; Fig. 2).

Recurrence rate in the follow-up. During follow-up, 3 cases $(15.0 \%)$ of recurrence were noted in the CIN I group, 5 cases (20.8\%) in the CIN II group and 9 cases $(30.0 \%)$ in the CIN III group. There was no significant differences in recurrence rate among the groups $(\mathrm{p}>0.05)$.

\section{Discussion}

This study showed galectin-3, VEGF-2 and cyclin D expression increased in patients with higher CIN stage classifications, but p $27^{\mathrm{kip} 1}$ showed the opposite trend. More than $80 \%$ of cervical 


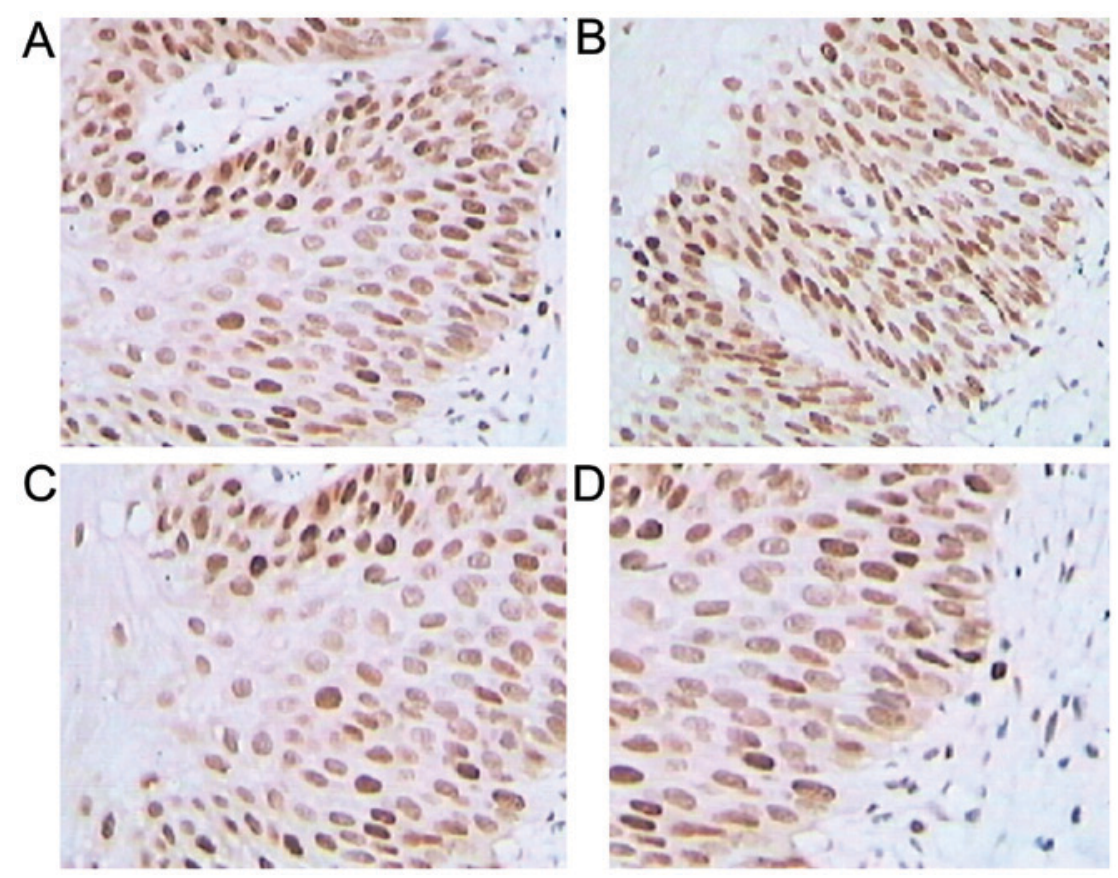

Figure 1. Immunohistochemical staining of CIN tissues (magnification, $\mathrm{x} 400$ ). (A) Positive galectin-3, total score of 6 points. (B) Positive p2 $7^{\mathrm{kip} 1}$, total score of 7 points. (C) Positive VEGF-2, total score of 8 points. (D) Positive cyclin D, total score of 9 points. CIN, cervical intraepithelial neoplasia; VEGF-2, vascular endothelial growth factor-2.

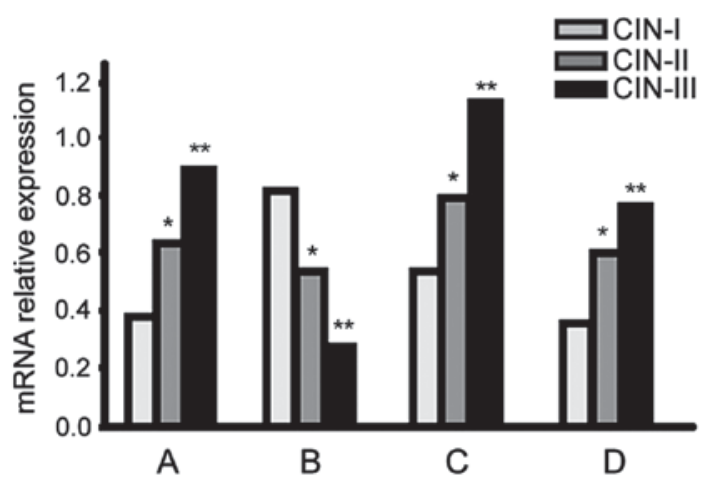

Figure 2. Detection of relative mRNA expression level of each protein in tissues via RT-PCR ( ${ }^{*}<<0.05$ between the CIN II and CIN I groups; ${ }^{* *} \mathrm{p}<0.05$ between the CIN III and CIN II groups; mRNA expression levels of galectin-3, VEGF-2 and cyclin D increased with increased CIN-staging, but $\mathrm{p} 27^{\mathrm{kipl}}$ showed the opposite trend. CIN, cervical intraepithelial neoplasia; VEGF-2, vascular endothelial growth factor-2.

cancer originates from CIN, so investigations regarding CIN tissue characteristics is extremely important for the study of cervical cancer occurrence. The galectin-3 gene is located on the human chromosome 14q21-22 and is comprised of a special chimeric structure composed of three parts: An N-terminal domain (with a serine phosphorylation site that regulates cell targeting), a collagen-like structure rich in repeating sequences of proline, glycine and tyrosine (as a matrix metalloproteinase substrate) and a C-terminal carbohydraterecognition domain (with a specific affinity for $\beta$-galactose residues) (11). Zhao et al (12) pointed out that galectin-3 can lead to conformational changes in mucoprotein 1 on the tumor surface, exposing the binding pockets of the adhesion molecule CD44 and its ligand and promote tumor cell adhesion and metastasis. Oka et al (13) confirmed that galectin-3 can inhibit tumor cell apoptosis induced by tumor necrosis factors such as CD95, resulting in tolerance to radiotherapy and chemotherapy. Peng et al (14) found that galectin-3 can induce $\mathrm{T}$ cell apoptosis and immune escape in vitro and in vivo and promote tumor proliferation. A number of studies (15) have argued that the positive expression of galectin-3 is related to pelvic lymph node metastasis and the poor prognosis of patients with cervical cancer. After VEGF binds to its receptor, signal transduction pathway activation results in the promotion of angiogenesis and lymphangiogenesis, providing a material basis for tumor growth and metastasis. Mitsuhashi et al (16) noted that the levels of serum VEGF and its receptors in patients with cervical squamous carcinoma were significantly higher than those in healthy controls, but no such differences were found in adenocarcinoma patients.

p $27^{\mathrm{kip} 1}$ is usually expressed in the nucleus of the middle-layer and superficial epithelial cells, but levels are lower or absent in basal-layer cells with active hyperplasia, suggesting that the $\mathrm{p} 27^{\mathrm{kipl}}$ protein functions by inhibiting cell division and promoting differentiation and maturation (17). HPV gene products release a large number of transcription factors in the G1 phase, activating gene expression required in the $\mathrm{S}$ phase and producing a variety of proteins required for the cell cycle including cyclin E. Cyclin E and p2 $7^{\mathrm{kip} 1}$ can bind to each other to induce the degradation of HPV gene products (18). Therefore, the downregulation of $\mathrm{p} 27^{\mathrm{kipl}}$ protein expression may be associated with HPV infection (19) or the result of cell cycle regulation disorders (20).

There was no difference in recurrence rate between groups during follow-up, but an increasing trend was noted, which may be related to the small sample size, shorter follow-up time and radical cure of lesion. In conclusion, the upregulation of galectin-3 expression and the downregulation of p2 $7^{\text {kipl }}$ expression in CIN tissues may be related to tumor progression 
and involving multiple mechanisms, such as angiogenesis, cell adhesion, apoptosis inhibition, immune escape, HPV infection and cell cycle regulation. This provides an important reference index for the early diagnosis and prognosis evaluation of cervical cancer, but its clinical application value needs to be further verified.

\section{References}

1. Xu XX, Zhou JS, Yuan SH, Yu H and Lou HM: Distribution of HPV genotype in invasive cervical carcinoma and cervical intraepithelial neoplasia in Zhejiang province, Southeast China: Establishing the baseline for surveillance. Int J Environ Res Public Health 12: 10794-10805, 2015.

2. Magaldi TG, Almstead LL, Bellone S, Prevatt EG, Santin AD and DiMaio D: Primary human cervical carcinoma cells require human papillomavirus E6 and E7 expression for ongoing proliferation. Virology 422: 114-124, 2012.

3. Than NG, Romero R, Balogh A, Karpati E, Mastrolia SA, Staretz-Chacham O, Hahn S, Erez O, Papp Z and Kim CJ: Galectins: Double-edged swords in the cross-roads of pregnancy complications and female reproductive tract inflammation and neoplasia. J Pathol Transl Med 49: 181-208, 2015.

4. Yu H, Zhang S, Zhang R and Zhang L: The role of VEGF-C/D and Flt-4 in the lymphatic metastasis of early-stage invasive cervical carcinoma. J Exp Clin Cancer Res 28: 98, 2009.

5. Markowska AI, Jefferies KC and Panjwani N: Galectin-3 protein modulates cell surface expression and activation of vascular endothelial growth factor receptor 2 in human endothelial cells. J Biol Chem 286: 29913-29921, 2011.

6. Punt S, Thijssen VL, Vrolijk J, de Kroon CD, Gorter A and Jordanova ES: Galectin-1, -3 and -9 expression and clinical significance in squamous cervical cancer. PLoS One 10: e0129119, 2015.

7. Ebrahim AH, Alalawi Z, Mirandola L, Rakhshanda R, Dahlbeck S, Nguyen D, Jenkins M, Grizzi F, Cobos E, Figueroa JA, et al: Galectins in cancer: Carcinogenesis, diagnosis and therapy. Ann Transl Med 2: 88, 2014.

8. Liu X, Yang WT and Zheng PS: Msi1 promotes tumor growth and cell proliferation by targeting cell cycle checkpoint proteins p21, p27 and p53 in cervical carcinomas. Oncotarget 5: 10870-10885, 2014.

9. Pavlides SC, Huang KT, Reid DA, Wu L, Blank SV, Mittal K, Guo L, Rothenberg E, Rueda B, Cardozo T, et al: Inhibitors of SCF-Skp2/Cks1 E3 ligase block estrogen-induced growth stimulation and degradation of nuclear p27kip1: Therapeutic potential for endometrial cancer. Endocrinology 154: 4030-4045, 2013.
10. Wu XL and Zheng PS: Undifferentiated embryonic cell transcription factor-1 (UTF1) inhibits the growth of cervical cancer cells by transactivating p27Kip1. Carcinogenesis 34: 1660-1668, 2013.

11. Kovak MR, Saraswati S, Schoen DJ and Diekman AB: Investigation of galectin-3 function in the reproductive tract by identification of binding ligands in human seminal plasma. Am J Reprod Immunol 72: 403-412, 2014

12. Zhao Q, Guo X, Nash GB, Stone PC, Hilkens J, Rhodes JM and Yu LG: Circulating galectin-3 promotes metastasis by modifying MUC1 localization on cancer cell surface. Cancer Res 69: 6799-6806, 2009.

13. Oka N, Nakahara S, Takenaka Y, Fukumori T, Hogan V, Kanayama HO, Yanagawa T and Raz A: Galectin-3 inhibits tumor necrosis factor-related apoptosis-inducing ligand-induced apoptosis by activating Akt in human bladder carcinoma cells. Cancer Res 65: 7546-7553, 2005.

14. Peng W, Wang HY, Miyahara Y, Peng G and Wang RF: Tumor-associated galectin-3 modulates the function of tumor-reactive T cells. Cancer Res 68: 7228-7236, 2008.

15. Wang W, Guo H, Geng J, Zheng X, Wei H, Sun R and Tian Z: Tumor-released Galectin-3, a soluble inhibitory ligand of human NKp30, plays an important role in tumor escape from NK cell attack. J Biol Chem 289: 33311-33319, 2014.

16. Mitsuhashi A, Suzuka K, Yamazawa K, Matsui H, Seki K and Sekiya S: Serum vascular endothelial growth factor (VEGF) and VEGF-C levels as tumor markers in patients with cervical carcinoma. Cancer 103: 724-730, 2005.

17. Cui N, Yang WT and Zheng PS: Slug inhibits the proliferation and tumor formation of human cervical cancer cells by up-regulating the $\mathrm{p} 21 / \mathrm{p} 27$ proteins and down-regulating the activity of the $\mathrm{Wnt} / \beta$-catenin signaling pathway via the trans-suppression Akt1/p-Akt1 expression. Oncotarget 7: 26152-26167, 2016.

18. Huang K-T, Pavlides SC, Lecanda J, Blank SV, Mittal KR and Gold LI: Estrogen and progesterone regulate p27kip1 levels via the ubiquitin-proteasome system: Pathogenic and therapeutic implications for endometrial cancer. PLoS One 7: e46072, 2012.

19. Zhou N, Yuan S, Wang R, Zhang W and Chen JJ: Role of dual specificity tyrosine-phosphorylation-regulated kinase 1B (Dyrk1B) in S-phase entry of HPV E7 expressing cells from quiescence. Oncotarget 6: 30745-30761, 2015.

20. Rath SL and Senapati S: Mechanism of p27 unfolding for CDK2 reactivation. Sci Rep 6: 26450, 2016.

This work is licensed under a Creative Commons Attribution-NonCommercial-NoDerivatives 4.0 International (CC BY-NC-ND 4.0) License. 